\title{
DISTAL FUNCTIONS AND UNIQUE ERGODICITY
}

\author{
EBRAHIM SALEHI
}

\begin{abstract}
A. Knapp [5] has shown that the set, $D(S)$, of all distal functions on a group $S$ is a norm closed subalgebra of $l^{\infty}(S)$ that contains the constants and is closed under the complex conjugation and left translation by elements of $S$. Also it is proved that [7] for any $k \in \mathbb{N}$ and any $\lambda \in \mathbb{R}$ the function $f: \mathbb{Z} \rightarrow \mathbb{C}$ defined by $f(n)=e^{i \lambda n^{k}}$ is distal on $\mathbb{Z}$. Now let $\mathbf{W}$ be the norm closure of the algebra generated by the set of functions

$$
\left\{n \mapsto e^{i \lambda n^{k}}: k \in \mathbb{N}, \lambda \in \mathbb{R}\right\},
$$

which will be called the Weyl algebra. According to the facts mentioned above, all members of the Weyl Algebra are distal functions on $\mathbb{Z}$. In this paper, we will show that any element of $\mathbf{W}$ is uniquely ergodic (Theorem 2.13) and that the set $\mathbf{W}$ does not exhaust all the distal functions on $\mathbb{Z}$ (Theorem 2.14). The latter will answer the question that has been asked (to the best of my knowledge) by $P$. Milnes [6].

The term Weyl algebra is suggested by S. Glasner. I would like to express my warmest gratitude to $S$. Glasner for his helpful advise, and to my advisor Professor Namioka for his enormous helps and contributions.
\end{abstract}

\section{Preliminaries}

Let $S$ be a semigroup and $X=l^{\infty}(S)$ be the algebra of all bounded complex functions on $S$, equiped with the topology of pointwise convergence on $S$. Then $\left(S, l^{\infty}(S)\right)$ forms a flow, where the action of $S$ on $X=l^{\infty}(S)$ is defined by

$$
(s, f) \mapsto s f=R_{s} f,
$$

where $(s f)(t)=\left(R_{s} f\right)(t)=f(t s)$ for all $f \in X$ and $s, t \in S$. A member $f \in l^{\infty}(S)$ is called a distal function on $S$ if it is, distal point relative to the flow $\left(S, l^{\infty}(S)\right)$. For this flow and its distal functions we have the following results $[7,8]$ :

1.1. Theorem. Let $S$ be a semigroup and let $\Sigma(X)$ be the enveloping semigroup of the flow $\left(S, l^{\infty}(S)\right)$. Then $\Sigma(X)$ is compact. Furthermore, each $\sigma \in \Sigma(X)$ is linear, multiplicative, and preserves the complex conjugation and the constant functions. Also $\|\sigma f\| \leq\|f\|$ holds for all $f \in l^{\infty}(S)$.

Received by the editors June 20, 1988 and, in revised form, January 31,1989 . Presented to the Society April 12, 1986 in Indianapolis, Indiana.

1980 Mathematics Subject Classification (1985 Revision). Primary 54H20; Secondary 28D99. 
1.2. Theorem. Let $D$ be the space of all distal functions on a semigroup $S$. Then $D$ is a norm closed subalgebra of $l^{\infty}(S)$ containing constants and is closed under complex conjugation. Furthermore, $D$ is invariant under each member $\sigma$ of the enveloping semigroup of the flow $\left(S, l^{\infty}(S)\right)$.

Given two flows $(S, X)$, and $(S, Y)$ a continuous function $\varphi: X \rightarrow Y$ is called a flow homomorphism if $\varphi(s x)=s \varphi(x)$ holds for all $s \in S$ and $x \in X$. If, in addition, $\varphi$ is onto (or 1-1), then it is called a flow epimorphism (or monomorphism). The following lemma facilitates a way to generate distal functions on $S$ from distal points of a flow $(S, X)$.

1.3. Lemma. Let $(S, X)$ be a flow, $F \in C(X)$, and $x_{0} \in X$ be a distal point, and let the function $f: S \rightarrow \mathbb{C}$ be defined by $f(s)=F\left(s x_{0}\right)$. Then $f$ is a distal on $S$.

Proof. Consider the mapping $\varphi: X \rightarrow l^{\infty}(S)$ defined by

$$
\varphi(x)(t)=F(t x) \text { for all } x \in X, t \in S,
$$

which is a flow homomorphism. Since the homomorphic image of a distal flow is again distal [2], $\varphi$ maps any distal point of $X$ to a distal point of $l^{\infty}(S)$. Thus, $f=\varphi\left(x_{0}\right)$ is a distal function on $S$.

A (classical) dynamical system is a pair $(X, T)$, where $X$ is a nonempty compact $T_{2}$ topological space, and $T$ is a continuous map of $X$ into itself. Note that this is precisely the flow $(\mathbb{N}, X)$ with the action

$$
(n, x) \mapsto T^{n} x,
$$

and if $T: X \rightarrow X$ is a homeomorphism, then it is the flow $(\mathbb{Z}, X)$ with the same action. Let $(X, T)$ and $(Y, S)$ be two dynamical systems. Then a continuous function $\varphi: X \rightarrow Y$ is called a homomorphism if $\varphi(T x)=S \varphi(x)$. If, in addition, $\varphi$ is onto, then it is called an epimorphism. A measure $\mu$ on $X$ is said to be invariant under $T$ if $\mu(f)=\mu(f \circ T)$ holds for all $f \in C(X)$. Following $\mathrm{H}$. Furstenberg [3] we shall refer to the triple $(X, T, \mu)$ as a process, where $(X, T)$ is a dynamical system, and $\mu$ is an invariant probability measure on the Borel subsets of $X$. For any $f \in C(X)$ we define an associated sequence $\left\{f_{n}\right\}$ of functions on $X$ by

$$
f_{n}(x)=\frac{1}{n+1} \sum_{m=0}^{n} f\left(T^{m} x\right) .
$$

A point $x \in X$ is said to be generic for the process $(X, T, \mu)$ if for any $f \in C(X)$ the sequence $\left\{f_{n}(x)\right\}$ converges to $\mu(f)$. A dynamical system $(X, T)$ is said to be uniquely ergodic if there is a unique invariant probability measure on the Borel subsets of $X$; if in addition, $X$ is minimal, then $(X, T)$ is called strictly ergodic.

The following theorem is a consequence of the Markov-Kakutani fixed point theorem [1, p. 45]. 
1.4. Theorem. For any dynamical system $(X, T)$ there is a probability measure $\mu$ on the Borel subsets of $X$ that is invariant under $T$.

And, for uniquely ergodic processes we have the following theorem [3, 9].

1.5. Theorem. Let $(X, T, \mu)$ be a process. Then the following are equivalent:

(a) $(X, T)$ is uniquely ergodic with invariant measure $\mu$;

(b) For any $f \in C(X)$ its associated sequence $\left\{f_{n}\right\}$ converges to $\mu(f)$ uniformly on $X$;

(c) Every point of $X$ is generic for the process $(X, T, \mu)$.

1.6. Corollary. Let $(X, T)$ be a dynamical system in which for any $f \in C(X)$ its associated sequence $\left\{f_{n}\right\}$ converges pointwise to a constant on $X$. Then $(X, T)$ is uniquely ergodic.

1.7. Corollary. Let $A$ be a dense linear subspace of $C(X)$, and assume that for any $f \in A$ its associated sequence $\left\{f_{n}\right\}$ converges pointwise to a constant. Then $(X, T)$ is uniquely ergodic.

1.8. Corollary. If the process $(X, T, \mu)$ is uniquely ergodic and $\varphi:(X, T) \rightarrow$ $(Y, S)$ is an epimorphism, then $(Y, S)$ is also uniquely ergodic.

\section{ERGODICITY OF ELEMENTS OF THE WEYL ALGEBRA}

In this section we would like to study the dynamical system $(X, S)$, where $X=l^{\infty}(\mathbb{Z})$, and $S: X \rightarrow X$ is the shift operator defined by $S f(n)=f(n+1)$. We note that this is exactly the flow $\left(\mathbb{Z}, l^{\infty}(\mathbb{Z})\right)$. For a function $f \in X$, let $X_{f}$ be the orbit closure of $f$, that is, the closure of the set $\left\{S^{n} f ; n \in \mathbb{Z}\right\}$. Since $\Sigma$, the enveloping semigroup of $\left(\mathbb{Z}, l^{\infty}(\mathbb{Z})\right)$, is compact, we have

$$
X_{f}=\Sigma f=\{\sigma(f): \sigma \in \Sigma\} .
$$

Also, if $f$ is distal, then the $X_{f}$ is minimal. Clearly, $S: X \rightarrow X$ is a homeomorphism, and $S\left(X_{f}\right)=X_{f}$. Thus the restriction of $S$ to $X_{f}$, which will be denoted by $S$, is still a homeomorphism, and we can consider the dynamical system $\left(X_{f}, S\right)$. By Theorem 1.4, there is a probability measure on the Borel subsets of $X_{f}$, which is invariant under $S$, and therefore invariant under all $S^{n}(n \in \mathbb{Z})$.

2.1. Definition. A function $f \in X$ is said to be uniquely ergodic if the system $\left(X_{f}, S\right)$ is uniquely ergodic. If in addition $X_{f}$ is also minimal, then we call $f$ strictly ergodic.

For a distal function $f$, since $X_{f}$ is minimal unique ergodicity is the same as strict ergodicity; therefore we do not make any distinction between them.

2.2. Theorem. Suppose that $f \in X$ is uniquely ergodic and that the function $F: \mathbb{C} \rightarrow \mathbb{C}$ is continuous. Then $F \circ f$ is uniquely ergodic.

Proof. Let $h=F \circ f$ and $X_{h}$ be the orbit closure of $h$, and let $\varphi: X_{f} \rightarrow X_{h}$ be defined by $\varphi(g)=F \circ g\left(g \in X_{f}\right)$. Then $\varphi$ is an epimorphism, and thus by Corollary $1.8, h$ is uniquely ergodic. 
2.3. Theorem. For a finite subset $\Omega=\left\{\omega_{1}, \omega_{2}, \ldots, \omega_{j}\right\}$ of $\mathbb{Z}$ let the functions $E(\Omega): X \rightarrow \mathbb{C}, \quad \bar{E}(\Omega): X \rightarrow \mathbb{C}$,

defined by

$$
\begin{gathered}
E(\Omega)(f)=f\left(\omega_{1}\right) f\left(\omega_{2}\right) \cdots f\left(\omega_{j}\right), \quad \text { and } \quad E(\varnothing)(f)=1, \\
\bar{E}(\Omega)(f)=\overline{E(\Omega)(f),}
\end{gathered}
$$

where $\overline{E(\Omega)(f)}$ is the complex conjugate of $E(\Omega)(f)$. Then $f$ is uniquely ergodic if and only if for any finite subsets $\Omega, \Delta$ of $\mathbb{Z}$,

$$
\frac{1}{n+1} \sum_{m=0}^{n} E(\Omega) \bar{E}(\Delta)\left(S^{m}(\sigma f)\right)
$$

converges, as $n \rightarrow \infty$, to a constant $c(\Omega, \Delta)$ that is independent of $\sigma \in \Sigma$.

Proof. $\Rightarrow$ Let $\varphi=E(\Omega) \bar{E}(\Delta)$. Then $\varphi^{\prime} \in C\left(X_{f}\right)$, and by $1.5(\mathrm{~b})$, its associated sequence $\left\{\varphi_{n}\right\}$ converges to $\mu(\varphi)$ uniformly on $X_{f}$.

$\Leftarrow$ Let $A$ be the linear subspace of $C\left(X_{f}\right)$ generated by all the functions of the form $E(\Omega) \bar{E}(\Delta)$ with $\Omega$ and $\Delta$ being any finite subsets of $\mathbb{Z}$. Then by the Stone-Weierstrass theorem, $A$ is dense in $C\left(X_{f}\right)$, and the theorem follows from Corollary 1.7.

2.4. Corollary. If $f$ is uniquely ergodic, then the limit

$$
\lim _{n} \frac{1}{n+1} \sum_{m=0}^{n}[\sigma(f)(m)]
$$

exists and is a constant independent of $\sigma$.

Proof. With the notation of the Theorem 2.3, let $\Omega=\{0\}$, and $\Delta=\varnothing$.

2.5. Corollary. If the function $f$ is uniquely ergodic, then so is $\tau f$ for any $\tau \in \Sigma$.

Proof. Let $\sigma \in \Sigma$, and $\nu=\sigma \circ \tau$. Then by Theorem 2.3,

$$
\frac{1}{n+1} \sum_{m=0}^{n} E(\Omega) \bar{E}(\Delta)\left(S^{m}(\sigma(\tau f))\right)=\frac{1}{n+1} \sum_{m=0}^{n} E(\Omega) \bar{E}(\Delta)\left(S^{m}(\nu f)\right),
$$

which converges uniformly to a constant independent of $\nu$.

2.6. Lemma. Let $p(x)=a_{k} x^{k}+a_{k-1} x^{k-1}+\cdots+a_{1} x+a_{0}$ be a real polynomial, and the function $f: \mathbb{Z} \rightarrow \mathbb{C}$ be defined by

$$
f(x)=e^{i p(x)} \quad(x \in \mathbb{Z}) .
$$

Then for each $\sigma \in \Sigma$ there is a real polynomial $p_{\sigma}$ whose leading coefficient is $a_{k}$, and $\sigma f(n)=e^{i p_{\sigma}(n)}$.

Proof. The conclusion follows obviously if $k=\operatorname{deg} p(x)=0$. So let $k>0$, and note that for any $n, s \in \mathbb{Z}$

$$
p(n+s)=p(n)+\frac{s}{1 !} p^{\prime}(n)+\frac{s^{2}}{2 !} p^{\prime \prime}(n)+\cdots+\frac{s^{k}}{k !} p^{(k)}(n) .
$$


Now suppose that $\left\{s_{\alpha}\right\}$ is a net in $\mathbb{Z}$ such that $\lim _{\alpha} s_{\alpha}=\sigma \in \Sigma$. By taking a subnet, if necessary, we may assume that

$$
\lim _{\alpha} \exp \left(i \frac{s^{l} \alpha}{l !}\right)=\exp \left(i \theta_{l}\right) \quad(l=1,2, \ldots, k)
$$

Consequently, for each $n \in \mathbb{Z}$

$$
\sigma f(n)=\lim _{\alpha} f\left(n+s_{\alpha}\right)=\lim _{\alpha} e^{i p\left(n+s_{\alpha}\right)}=e^{i p_{\sigma}(n)},
$$

where $p_{\sigma}(n)=p(n)+\theta_{1} p^{\prime}(n)+\theta_{2} p^{\prime \prime}(n)+\cdots+\theta_{k} p^{(k)}(n)$ is a real polynomial in $n$, whose leading coefficient is the same as that of $p(n)$, i.e., $a_{k}$.

2.7. Lemma. Let $F: \mathbb{Z} \rightarrow \mathbb{C}$ be a periodic function with period $u>0$, and $p(x)=a_{q} x^{q}+a_{q-1} x^{q-1}+\cdots+a_{1} x+a_{0}$ be a real polynomial for which, if $q>0$, the leading coefficient $a_{q}$ is an irrational multiple of $\pi$. Then

$$
\lim _{n \rightarrow \infty}\left[\frac{1}{n+1} \sum_{m=0}^{n} F(m) e^{i p(m)}\right]= \begin{cases}0, & \text { if } q>0, \\ \frac{e^{i a_{0}}}{u}[F(0)+\cdots+F(u-1)], & \text { if } q=0 .\end{cases}
$$

Proof. Observe that, for any $n \in \mathbb{N}$, there is a nonnegative integer $k$ with $n=k u+r \quad(0 \leq r<u)$. Hence

$$
\begin{aligned}
\frac{1}{n+1} \sum_{m=0}^{n} F(m) e^{i p(m)}= & \frac{k u}{k u+r+1} \cdot \frac{1}{k u} \sum_{m=0}^{k u-1} F(m) e^{i p(m)} \\
& +\frac{1}{n+1} \sum_{m=k u}^{n} F(m) e^{i p(m)} .
\end{aligned}
$$

If $F(m) e^{i p(m)}$ is bounded by $M$, then $\left|\sum_{m=k u}^{n} F(m) e^{i p(m)}\right| \leq u M$. Therefore,

$$
\lim _{n \rightarrow \infty}\left[\frac{1}{n+1} \sum_{m=k u}^{n} F(m) e^{i p(m)}\right]=0
$$

Also, $\lim _{k \rightarrow \infty} k u /(k u+r+1)=1$. Thus it is sufficient to calculate

$$
\lim _{k \rightarrow \infty}\left[\frac{1}{k u} \sum_{m=0}^{k u-1} F(m) e^{i p(m)}\right]
$$


But

$$
\begin{aligned}
\sum_{m=0}^{k u-1} F(m) e^{i p(m)} & =\sum_{j=0}^{k-1} \sum_{m=j u}^{(j+1) u-1} F(m) e^{i p(m)} \\
& =\sum_{j=0}^{k-1} \sum_{m=0}^{u-1} F(m+j u) e^{i p(m+j u)} \\
& =\sum_{j=0}^{k-1} \sum_{m=0}^{u-1} F(m) e^{i p(m+j u)} \\
& =\sum_{m=0}^{u-1} \sum_{j=0}^{k-1} F(m) e^{i p(m+j u)} \\
& =\sum_{m=0}^{u-1} F(m) \sum_{j=0}^{k-1} e^{i p(m+j u)} .
\end{aligned}
$$

If $q=\operatorname{deg} p(x)=0$, then $e^{i p(m+j u)}=e^{i a_{0}}$, and

$$
\begin{aligned}
\frac{1}{k u} \sum_{m=0}^{k u-1} F(m) e^{i p(m)} & =\frac{1}{k u} \sum_{m=0}^{u-1} F(m) \sum_{j=0}^{k-1} e^{i p(m+j u)} \\
& =\frac{1}{k u} \sum_{m=0}^{u-1} F(m) \sum_{j=0}^{k-1} e^{i a_{0}} \\
& =\frac{e^{i a_{0}}}{u}[F(0)+\cdots+F(u-1)] .
\end{aligned}
$$

For $q>0$, note that $p(m+j u)$ is a polynomial in $j$, whose leading coefficient, $a_{q} u^{q}$, is an irrational multiple of $\pi$. Then, by the theorem of Weyl [10, Satz 9]

$$
\lim _{k \rightarrow \infty}\left[\frac{1}{k} \sum_{j=0}^{k-1} e^{i p(m+j u)}\right]=0
$$

Consequently

$$
\lim _{k \rightarrow \infty}\left[\frac{1}{k u} \sum_{m=0}^{k u-1} F(m) e^{i p(m)}\right]=\sum_{m=0}^{u-1} F(m)\left\{\lim _{k \rightarrow \infty}\left[\frac{1}{k u} \sum_{j=0}^{k-1} e^{i p(m+j u)}\right]\right\}=0,
$$

and this proves the lemma.

2.8. Theorem. Let $k \in \mathbb{N}$ be fixed and let

$$
p(x)=a_{k} x^{k}+a_{k-1} x^{k-1}+\cdots+a_{1} x+a_{0}
$$

be a real polynomial of degree $k$. Then the function $h: \mathbb{Z} \rightarrow \mathbb{C}$ defined by $h(x)=e^{i p(x)}$ is uniquely ergodic.

Proof. If $a_{1}, a_{2}, \ldots, a_{k}$, are all rational multiplies of $\pi$, then $h$ is periodic and $X_{h}$ is finite and minimal. Hence it is uniquely ergodic. So, we may assume 
that at least one of the coefficients $a_{1}, a_{2}, \ldots, a_{k}$, is an irrational multiple of $\pi$. Suppose that $a_{q}(0<q \leq k)$ is the last such number, and let

$$
\begin{aligned}
& Q(n)=a_{k} x^{k}+\cdots+a_{q+1} x^{q+1}, \\
& R(n)=a_{q} x^{q}+\cdots+a_{1} x+a_{0} .
\end{aligned}
$$

If $f(n)=e^{i Q(n)}$ and $g(n)=e^{i R(n)}$, then $h(n)=f(n) g(n)$, and the function $f$ is a periodic function (say, with period $u$ ). By Lemma 2.6, for any $\sigma$, there is a real polynomial $R_{\sigma}$ with the same leading coefficient as that of $R$ (i.e., $a_{q}$ ), such that $\sigma g(n)=e^{i R_{\sigma}(n)}$. Now let $\Omega=\left\{\omega_{1}, \omega_{2}, \ldots, \omega_{j}\right\}$, and $\Delta=\left\{\delta_{1}, \delta_{2}, \ldots, \delta_{l}\right\}$ be any two finite subsets of $\mathbb{Z}$. Then by Theorem 2.3 , we must show that

$$
\lim _{n \rightarrow \infty}\left[\frac{1}{n+1} \sum_{m=0}^{n} E(\Omega) \bar{E}(\Delta)\left(S^{m}(\sigma h)\right)\right]
$$

exists and its value is independent of $\sigma$. Using the properties of $\sigma, S, E$, and $\bar{E}$ we note that

$$
\begin{aligned}
E(\Omega) \bar{E}(\Delta)\left(S^{m} \sigma h\right) & =E(\Omega) \bar{E}(\Delta)\left(S^{m}(\sigma f \sigma g)\right) \\
& =\left[E(\Omega) \bar{E}(\Delta)\left(S^{m}(\sigma f)\right)\right] \cdot[E(m+\Omega) \bar{E}(m+\Delta)(\sigma g)] \\
& =\left[E(\Omega) \bar{E}(\Delta)\left(S^{m}(\sigma f)\right)\right] \cdot\left[E(m+\Omega) \bar{E}(m+\Delta) e^{i R_{\sigma}}\right] \\
& \left.=E(\Omega) \bar{E}(\Delta)\left(S^{m}(\sigma f)\right)\right] \cdot e^{i G_{\sigma}(m)},
\end{aligned}
$$

where $G_{\sigma}(m)=\sum_{\omega \in \Omega} R_{\sigma}(m+\omega)-\sum_{\delta \in \Delta} R_{\sigma}(m+\delta)$ is a polynomial in $m$. Now let $F: \mathbb{Z} \rightarrow \mathbb{C}$ be defined by

$$
F(m)=E(\Omega) \bar{E}(\Delta)\left(S^{m} f\right) .
$$

Then $F$ is periodic function of period $u$, and a straightforward calculation shows that

Consequently,

$$
E(\Omega) \bar{E}(\Delta)\left(S^{m} \sigma f\right)=(\sigma F)(m)
$$

$$
\frac{1}{n+1} \sum_{m=0}^{n} E(\Omega) \bar{E}(\Delta)\left(S^{m}(\sigma h)\right)=\frac{1}{n+1} \sum_{m=0}^{n}(\sigma F)(m) e^{i G_{\sigma}(m)} .
$$

Also, if $\left\{s_{\alpha}\right\}$ is a net in $\mathbb{Z}$ such that $\lim _{\alpha} s_{\alpha}=\sigma \in \Sigma$, then

$$
\begin{gathered}
\sigma F(0)+\cdots+\sigma F(u-1)=\lim _{\alpha}\left[F\left(0+s_{\alpha}\right)+\cdots+F\left(u-1+s_{\alpha}\right)\right] \\
\quad=\lim _{\alpha}[F(0)+\cdots+F(u-1)]=F(0)+\cdots+F(u-1),
\end{gathered}
$$

which shows that $\sigma F(0)+\cdots+\sigma F(u-1)$ is a constant independent of $\sigma \in \Sigma$. Similarly, one can show that $\sigma F$ is also a periodic function of period $u$. On the other hand, by Taylor's theorem

$$
\begin{aligned}
G_{\sigma}(m) & =\sum_{\omega \in \Omega} R_{\sigma}(m+\omega)-\sum_{\delta \in \Delta} R_{\sigma}(m+\delta) \\
& =\lambda_{0} R_{\sigma}(m)+\lambda_{1} R_{\sigma}^{\prime}(m)+\cdots+\lambda_{q} R_{\sigma}^{(q)}(m),
\end{aligned}
$$


where $\lambda_{\nu}=\frac{1}{\nu !}\left(\omega_{1}^{\nu}+\cdots+\omega_{j}^{\nu}-\delta_{1}^{\nu}-\cdots-\delta_{l}^{\nu}\right) \quad(\nu=0,1, \ldots, q)$ is a rational number independent of $\sigma$. 2.7,

Now, if $\lambda_{0}=\lambda_{1}=\cdots=\lambda_{q-1}=0$, then $G_{\sigma}(m)=\lambda_{q} a_{q} q$ ! and, by Lemma

$$
\begin{gathered}
\lim _{n \rightarrow \infty}\left[\frac{1}{n+1} \sum_{m=0}^{n}(\sigma F)(m) e^{i G_{\sigma}(m)}\right]=\frac{e^{i \lambda_{q} a_{q} q !}}{u}[\sigma F(0)+\cdots+\sigma F(u-1)] \\
=\frac{e^{i \lambda_{q} a_{q} q !}}{u}[F(0)+\cdots+F(u-1)],
\end{gathered}
$$

which is independent of $\sigma$. If one of $\lambda_{0}, \lambda_{1}, \ldots, \lambda_{q-1}$ is nonzero, say $\lambda_{r} \neq 0$, but $\lambda_{0}=\lambda_{1}=\cdots=\lambda_{r-1}=0 \quad(0 \leq r<q)$, then $\operatorname{deg} G_{\sigma}(m)=r$, and its leading coefficient is the same as that of $\lambda_{r} R_{\sigma}^{(r)}(m)$, which is an irrational multiple of $\pi$. Therefore, by Lemma 2.7,

$$
\lim _{n \rightarrow \infty}\left[\frac{1}{n+1} \sum_{m=0}^{n}(\sigma F)(m) e^{i G_{\sigma}(m)}\right]=0 .
$$

Thus, in either case, the limit

$$
\lim _{n \rightarrow \infty}\left[\frac{1}{n+1} \sum_{m=0}^{n} E(\Omega) \bar{E}(\Delta)\left(S^{m} \sigma h\right)\right]=\lim _{n \rightarrow \infty}\left[\frac{1}{n+1} \sum_{m=0}^{n}(\sigma F)(m) e^{i G(m)}\right]
$$

exists and is independent of $\sigma \in \Sigma$. This completes the proof.

2.9. Theorem. Let the system $(X, T)$ be strictly ergodic, with $T: X \rightarrow X$ a homeomorphism, and let the function $\Lambda: C(X) \times X \rightarrow l^{\infty}(\mathbb{Z})$ be defined by $\Lambda(F, x)(n)=F\left(T^{n} x\right)$. Then any element in the range of $\Lambda$ is strictly ergodic. Proof. Let $f=\Lambda\left(F, x_{0}\right)$ be fixed, and define the mapping $\varphi: X \rightarrow l^{\infty}(\mathbb{Z})$ by $\varphi(x)(n)=F\left(T^{n} x\right)$. Then $\varphi\left(x_{0}\right)=f$, and

$$
\varphi(T x)(n)=F\left(T^{n}(T x)\right)=F\left(T^{n+1} x\right)=\varphi(x)(n+1)=(S \varphi(x))(n)
$$

or $\varphi(T x)=S \varphi(x)$, which shows that $\varphi$ is a flow homomorphism. Since $X$ is minimal, $\varphi[X]=X_{f}$, the orbit closure of $f$. Therefore, by Corollary 1.7, $f$ is strictly ergodic.

Next we would like to show that any function $h: \mathbb{Z} \rightarrow \mathbb{C}$ of the form $h(n)=\sum_{j=1}^{r} A_{j} e^{i P_{j}(n)}$ is uniquely ergodic, where $A_{j} \in \mathbb{C}$ and $P_{j}(n)$ is a real polynomial. The proof is presented for the case $r=2$, but it can be applied to any $r \in \mathbb{N}$. First we need the following theorem.

2.10. Theorem. Let $f, g$ be two uniquely ergodic functions on $\mathbb{Z}$ such that for any four finite subsets $\Omega_{1}, \Omega_{2}, \Delta_{1}$, and $\Delta_{2}$ of $\mathbb{Z}$, the function

$$
F\left(\Omega_{1}, \Omega_{2}, \Delta_{1}, \Delta_{2}\right)=\prod_{\omega \in \Omega_{1}} S^{\omega} f \prod_{\omega \in \Omega_{2}} S^{\omega} g \prod_{\delta \in \Delta_{1}} S^{\delta \bar{f}} \prod_{\delta \in \Delta_{2}} S^{\delta} \bar{g}
$$

is uniquely ergodic. Then $f+g$ is uniquely ergodic. 
Proof. First we consider $Y=\Sigma(f, g)$, the orbit closure of $(f, g)$, and we wish to show that it is uniquely ergodic. By Corollary 1.6, we must prove that for any $\varphi \in C(\Sigma(f, g))$, and any element $\sigma(f, g)=(\sigma f, \sigma g) \in \Sigma(f, g)$ the limit

$$
\lim _{n \rightarrow \infty}\left[\frac{1}{n+1} \sum_{m=0}^{n} \varphi\left(S^{m}(\sigma f, \sigma g)\right)\right]
$$

exists and is a constant independent of $\sigma$. For this we use the fact that $\Sigma(f, g) \subset \Sigma f \times \Sigma g=X_{f} \times X_{g}$. Following the notation of Theorem 2.3, for a finite subset $\Omega$ of $\mathbb{Z}$, let the functions

$$
E_{1}(\Omega): \Sigma f \times \Sigma g \rightarrow \mathbb{C}, \quad E_{2}(\Omega): \Sigma f \times \Sigma g \rightarrow \mathbb{C}
$$

be defined by $E_{1}(\Omega)(\sigma f, \tau g)=E(\Omega)(\sigma f)$, and $E_{2}(\Omega)(\sigma f, \tau g)=E(\Omega)(\tau g)$, and let $\overline{E_{j}}(\Omega)$ be the complex conjugate of $E_{j}(\Omega), j=1,2$. Then $E_{j}(\Omega)$ is continuous and the set of linear combinations of the functions of the form

$$
E_{1}\left(\Omega_{1}\right) E_{2}\left(\Omega_{2}\right) \overline{E_{1}}\left(\Delta_{1}\right) \overline{E_{2}}\left(\Delta_{2}\right)
$$

is dense in $C(\Sigma f \times \Sigma g)=C\left(X_{f} \times X_{g}\right)$. Thus by Corollary 1.7, is enough to consider $\varphi=E_{1}\left(\Omega_{1}\right) E_{2}\left(\Omega_{2}\right) \overline{E_{1}}\left(\Delta_{1}\right) \overline{E_{2}}\left(\Delta_{2}\right)$. With this choice

$$
\varphi\left(S^{m}(\sigma f, \sigma g)\right)=F\left(\Omega_{1}, \Omega_{2}, \Delta_{1}, \Delta_{2}\right)(m) .
$$

Since $F\left(\Omega_{1}, \Omega_{2}, \Delta_{1}, \Delta_{2}\right)(m)$ is uniquely ergodic, by Theorem 2.3 , the limit

$$
\lim _{n}\left[\frac{1}{n+1} \sum_{m=0}^{n} \varphi\left(S^{m}(\sigma f, \sigma g)\right)\right]
$$

exists and is independent of $\sigma$. This proves that $Y=\Sigma(f, g)$ is uniquely ergodic. Next by Theorem 2.9, the mapping $\Lambda: C(Y) \times Y \rightarrow l^{\infty}(\mathbb{Z})$ defined by $\Lambda(G, y)(n)=G\left(S^{n} y\right)$ produces uniquely ergodic functions. Now let $y_{0}=(f, g)$, and $G=E_{1}(0)+E_{2}(0)$. Then

$$
\Lambda\left(G, y_{0}\right)(n)=G\left(S^{n} y_{0}\right)=G\left(S^{n}(f, g)\right)=G\left(S^{n} f, S^{n} g\right)=f(n)+g(n)
$$

is uniquely ergodic.

2.11. Corollary. Let $p(n), q(n)$ be any two real polynomials, and $f(n)=e^{i p(n)}$, and $g(n)=e^{i q(n)}$. Then $f+g$ is uniquely ergodic.

Proof. It is sufficient to show that, for any four finite subsets $\Omega_{1}, \Omega_{2}, \Delta_{1}$, and $\Delta_{2}$ of integers the function

$$
F\left(\Omega_{1}, \Omega_{2}, \Delta_{1}, \Delta_{2}\right)=\prod_{\omega \in \Omega_{1}} S^{\omega} f \prod_{\omega \in \Omega_{2}} S^{\omega} g \prod_{\delta \in \Delta_{1}} S^{\delta} \bar{f} \prod_{\delta \in \Delta_{2}} S^{\delta} \bar{g}
$$

is uniquely ergodic. But $F\left(\Omega_{1}, \Omega_{2}, \Delta_{1}, \Delta_{2}\right)(n)=e^{i Q(n)}$, where

$$
Q(n)=\sum_{\omega \in \Omega_{1}} p(n+\omega)+\sum_{\omega \in \Omega_{2}} q(n+\omega)-\sum_{\delta \in \Delta_{1}} p(n+\delta)-\sum_{\delta \in \Delta_{2}} q(n+\delta)
$$


is a real polynomial in $n$. Therefore, by Theorem 2.8, $F\left(\Omega_{1}, \Omega_{2}, \Delta_{1}, \Delta_{2}\right)$ is uniquely ergodic.

So far, it has been shown that any element of the algebra $\mathbf{A}$ generated by the set $\left\{n \mapsto e^{i \lambda n^{k}}: \lambda \in \mathbb{R}, k \in \mathbb{N}\right\}$ is strictly ergodic. Next, we would like to demonstrate the main result of this paper; that is, any element of the Weyl algebra $\mathbf{W}$ is strictly ergodic. To accomplish this result, we need the following theorem.

2.12. Theorem. The set of all uniquely ergodic functions in $X=l^{\infty}(\mathbb{Z})$ is closed under uniform convergence.

Proof. Let $f_{\alpha} \in X$ be a sequence of uniquely ergodic functions that converge uniformly to $f$. We choose $\Omega, \Delta$ to be any two finite subsets of $\mathbb{Z}$, and define the function $F: l^{\infty}(\mathbb{Z}) \rightarrow \mathbb{C}$ by $F(f)=E(\Omega) \bar{E}(\Delta)(f)$. For each $\alpha$, the function $f_{\alpha}$ is uniquely ergodic, and therefore there is a number $c_{\alpha}$ independent of $\sigma \in \Sigma$ such that

$$
\begin{aligned}
\lim _{n \rightarrow \infty} & {\left[\frac{1}{n+1} \sum_{m=0}^{n} E(\Omega) E(\Delta)\left(S^{m}\left(\sigma f_{\alpha}\right)\right)\right] } \\
& =\lim _{n \rightarrow \infty}\left[\frac{1}{n+1} \sum_{m=0}^{n} F\left(S^{m} \sigma f_{\alpha}\right)\right]=c_{\alpha} .
\end{aligned}
$$

Let $M=\sup _{\alpha}\left\|f_{\alpha}\right\|_{\infty}$. Then $S^{m}\left(\sigma\left(f_{\alpha}\right)\right)$ and $S^{m}(\sigma(f))$ are in the set

$$
B=\left\{h \in l^{\infty}(\mathbb{Z}):\|h\|_{\infty} \leq M\right\} .
$$

Since $F$ is uniformly continuous on $B,\left\{c_{\alpha}\right\}$ is Cauchy, and it converges to a number $c \in \mathbb{C}$. Now we would like to show that for any $\sigma \in \Sigma$

$$
\lim _{n \rightarrow \infty}\left[\frac{1}{n+1} \sum_{m=0}^{n} F\left(S^{m}(\sigma f)\right)\right]=c .
$$

Given $\varepsilon>0$, by uniform continuity of $F$ on $B$, there is a $\delta>0$ such that

$$
h_{1}, h_{2} \in B \text { and }\left\|h_{1}-h_{2}\right\|<\delta \Rightarrow\left|F\left(h_{1}\right)-F\left(h_{2}\right)\right|<\varepsilon / 3 .
$$

Let $\alpha$ be chosen so that $\left|c_{\alpha}-c\right|<\varepsilon / 3$, and $\left\|f_{\alpha}-f\right\|_{\infty}<\delta$, which implies that $\left|F\left(f_{\alpha}\right)-F(f)\right|<\varepsilon / 3$. Therefore

$$
\begin{aligned}
\left|\frac{1}{n+1} \sum_{m=0}^{n} F\left(S^{m}(\sigma f)\right)-c\right| \leq & \frac{1}{n+1} \sum_{m=0}^{n}\left|F\left(S^{m}(\sigma f)\right)-F\left(S^{m}\left(\sigma f_{\alpha}\right)\right)\right| \\
& +\left|\frac{1}{n+1} \sum_{m=0}^{n} F\left(S^{m}\left(\sigma f_{\alpha}\right)\right)-c_{\alpha}\right|+\left|c_{\alpha}-c\right| .
\end{aligned}
$$

Now, since $f_{\alpha}$ is uniquely ergodic, there is a number $M_{0} \in \mathbb{N}$ such that

$$
n \geq M_{0} \Rightarrow\left|\frac{1}{n+1} \sum_{m=0}^{n} F\left(S^{m}\left(\sigma f_{\alpha}\right)\right)-c_{\alpha}\right|<\frac{\varepsilon}{3} ;
$$


therefore

$$
n \geq M_{0} \Rightarrow\left|\frac{1}{n+1} \sum_{m=0}^{n} F\left(S^{m}(\sigma f)\right)-c\right|<\varepsilon,
$$

which completes the proof.

2.13. Theorem. Any element of the Weyl algebra is uniquely ergodic.

Proof. This follows from Theorem 2.12 and the fact that the Weyl algebra is the uniform closure of a set of uniquely ergodic functions.

2.14. Theorem. The Weyl algebra does not exhaust all distal functions.

Proof. By Theorem 2.13, it is enough to introduce a distal function on $\mathbb{Z}$ that is not uniquely ergodic. Let $\mathbb{T}=\{z \in \mathbb{C}:|z|=1\}$ be the unit circle, $\lambda \in \mathbb{T}$, and $g: \mathbb{T} \rightarrow \mathbb{T}$ be any continuous function, and let $\varphi: \mathbb{T}^{2} \rightarrow \mathbb{T}^{2}$ be defined by

$$
\varphi(u, v)=(\lambda u, g(u) v) .
$$

Then the flow $\left(\mathbb{Z}, \mathbb{T}^{2}\right)$ is distal, where the action is $(n, \xi) \mapsto \varphi^{n}(\xi)$ [4]. In [3], Furstenburg has introduced a continuous function $g: \mathbb{T} \rightarrow \mathbb{T}$ and an element $\lambda \in \mathbb{T}$ such that not all ergodic averages do exist; that is, there is a $\xi \in \mathbb{T}^{2}$ and $h \in C\left(\mathbb{T}^{2}\right)$ such that

$$
\lim _{n \rightarrow \infty}\left[\frac{1}{n+1} \sum_{m=0}^{n} h\left(\varphi^{m}(\xi)\right)\right]
$$

does not exist. Now if we define $f: \mathbb{Z} \rightarrow \mathbb{C}$ by $f(m)=h\left(\varphi^{m}(\xi)\right)$, then, by Lemma 1.3, $f$ is distal, while, by Corollary 2.4 (with $\sigma=R_{0}$, the right translation by 0$)$; it is not uniquely ergodic $(f \notin \mathbf{W})$.

\section{REFERENCES}

1. J. R. Brown, Ergodic theory and topological dynamics, Academic Press, New York, 1976.

2. Robert Ellis, Lectures on topological dynamics, Benjamin, New York, 1969.

3. H. Furstenberg, Strict ergodicity and transformation of the torus, Amer. J. Math. 83 (1961), 573-601.

4. _ The structure of distal flow, Amer. J. Math. 85 (1963), 477-515.

5. A. W. Knapp, Distal functions on groups, Trans. Amer. Math. Soc. 128 (1967), 1-40.

6. Paul Milnes, Minimal and distal functions on a semidirect products of groups, Preprint.

7. Isaac Namioka, Affine flows and distal points, Math. Z. 184 (1983), 259-269.

8. _ Right topological groups, distal flows and a fixed point theorem, Math. Systems Theory 6 (1972), 193-209.

9. J. C. Oxtoby, Ergodic sets, Bull. Amer. Math. Soc. 58 (1952), 116-136.

10. H. Weyl, Über die Gleichverteilung von Zahlen mod eins, Math. Ann. 77 (1916), 313-352.

Department of Mathematics, University of Nevada, Las Vegas, Nevada 89154 